Pasadena) is also a potentially useful tool although no quantitative measurements of the coronal field have yet been determined. The Razin effect in solar radio emission (Boischot and Clavelier, Paris; Ramaty and Lingenfelter, Goddard; Bohlin and Simon, California Institute of Technology) appears to have given us the most unambiguous determination of the field at a value of 0.3 to $0.5 \mathrm{G}$ at $2 R_{\odot}$ above an active region. All these researches refer to the observation of a single Type IV burst and similar observations of other events is urgently required.

The connection of coronal magnetic fields to the density structure of the corona has been attacked from two directions. In the first, the surface distribution of the fields over the entire sun is used to calculate the fields in the corona; the shapes of the field lines are then compared to the visual appearance of the corona (Newkirk, Altschuler and Harvey, HAO, Boulder; Schatten, University of California). Although there is always some question about the validity of such current-free calculations in the corona, the correspondence between the shapes of the field lines and the small scale density structures in the corona such as arches and rays suggests that much of the small scale structure is due to the filling of magnetic tubes by various amounts of coronal material. These same analyses suggest that coronal streamers, indeed, form over extended regions of opposite polarity on the surface of the sun. Such a picture makes the apparent association of streamers with filaments a quite natural consequence. A similar conclusion was reached by Sturrock and Smith (Stanford) but without the benefit of a quantitative model of the coronal magnetic fields.

A second approach to the role played by magnetic fields in forming coronal streamers has involved the detailed solution of the hydromagnetic equations for the solar wind with a simple distribution of magnetic fields on the surface of the sun (Pneuman and Kopp, HAO, Boulder). These models predict rather well the shape of the streamers, and the density distribution within and outside of a streamer. The calculations may be extended to $1 \mathrm{AU}$ where it is found that the streamer represents a small increase in the local density and velocity over the background corona.

The question of the extension of the corona and its magnetic fields out into the interplanetary medium has also received considerable attention. Although it is found that a correlation exists between the fields calculated for the corona at 1.6 $R_{\odot}$ and those at $1 \mathrm{AU}$ (Schatten, Wilcox and Ness, University of California and Goddard) there still exists considerable disagreement as to whether the "mapping" or the "nozzle" hypothesis is a better interpretation of the data. At the present time, the connection between such visible structures in the corona as streamers and the features seen in the particles and fields at $1 \mathrm{AU}$ is largely unknown.

R. G. ATHAY

Vice-President of the Commission

\title{
WORKING GROUP ON CENTRAL LINE INTENSITIES
}

Of a select list of about 40 Fraunhofer lines distributed between $\lambda \lambda 3083-7122$, central intensities corrected for scattered light, ghosts and instrumental profile, has been obtained for 18 lines. Uncorrected values of central intensity for a number of other lines are available. A complete list of the results of all observers will be given at the General Assembly in Brighton.

A. KEITH PIERCE

Chairman of the Working Group

\section{WORKING GROUP ON SOLAR ECLIPSES}

The working Group on Solar Eclipses regrets Dr J. Houtgast's resignation as Chairman of the Group. However, Dr Houtgast will maintain his membership and continue to give his experience to the group.

The total eclipse of September 22, 1968 was actively observed by many groups in spite of its short duration. Reports on the observations are expected to be given during the 1970 IAU General Assembly.

A great deal of work has been done everywhere in view of the total eclipse of March 7, 1970. 
The National Science Foundation has issued a Solar Eclipse 1970 Bulletin which has been widely distributed in order to help the observers in making their plans and to coordinate the observations. Dr Albert E. Belon, U.S. Coordinator for the 1970 Solar Eclipse, has devoted to this heavy task all his attention and care.

The U.S. Naval Observatory published a circular with details on the circumstances and relevant data on the 1970 total eclipse, which has regularly distributed.

The Working Group on Solar Eclipse has been in contact with the U.S. Coordinator and with Dr Edward R. Dyer, Jr., Secretary of the IUCSTP for a mutual cooperation in exchanging information about the organization of the researches and observations to be performed during the 1970 solar eclipse.

A comprehensive report about the activity of the group and about the work performed during the solar eclipses of 1968 and 1970 will be prepared after the coming IAU General Assembly. 